\title{
books
}

\section{More than the sum of all parts}

\section{Jürgen Tautz}

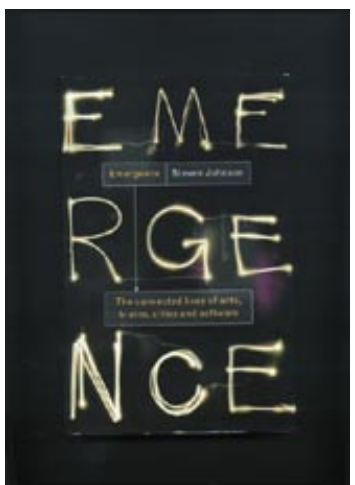

Emergence

by Steven Johnson

Allen Lane, Penguin Press, London, UK

288 pages, €24.60

ISBN 0713994002

What do slime moulds, ants, brains and cities have in common? According to the American media and technology critic Steven Johnson, they all display 'emergence', and he sets out to explain this mysterious-sounding term in this entertaining book. It all revolves around the well-known phenomenon that, in complex structures, the whole is often greater than the sum of its parts. How come large cities seem to be guided by an invisible hand as they 'learn' how to function optimally? And why do more thriving neighbourhoods spontaneously develop the less architectural planning takes place? Is it not astonishing that ants, hopelessly lost as individuals, collectively find the best solutions for problems imposed upon them by an unpredictable, complex and changing environment? And that bundles of stupid nerve cells, which function according to physical rules, develop into an intelligent brain that ultimately reaches self-awareness? The book takes on these and many more stimulating questions, and Johnson shows that a small number of rules can create 'global wisdom' in complex systems. In detail, these rules are: (i) take a huge number of individuals, (ii) make each of these individuals relatively simple, (iii) let them interact on a random basis and (iv) tell them to pay attention to their neighbours.

These rules are easy to understand if we apply them to systems that obviously demonstrate emergence. An individual ant has a 'micro-performance', but a colony exhibits a 'macro-behaviour', which cannot be predicted on the basis of observing a single organism. The entire system has to be observed at work to discover the global behaviour. Ten thousand 'dumb' ants collectively become smart, calculating the shortest routes to food supplies without any choreographer who can see the bigger picture. This has been attributed to 'swarm logic', a strategy now successfully applied in designing computers. And swarm logic is also the basis of brain construction where densely connected neurons lead to a sophisticated behaviour that finally results in intelligence. Decentralised systems such as these depend on random interactions. Each interaction is arbitrary but, as there are so many, the individual can gauge and filter them and eventually alter the macro-state of the system. Johnson explains this by extensively citing Jane Jacob's book, Death and Life of the Great American Cities. Meeting people face-to-face on the streets forms the gap-junctions of city life; cities that are car-centred offer limited potential for local interactions between their inhabitants and thus no higher-order level of urban life can emerge. I am sure that everyone has been in such a city and noticed the prevailing 'cemetery' atmosphere.

Johnson leads us on a journey from the 12th Century silk weaver district in medieval Florence via early industrialised Manchester to ant colonies, brains and ultimately to the world-wide web. He neatly spans the gaps between these seemingly disparate examples to explain how 21 st Century cities are not that much different from ant colonies in 'learning' how to react to new challenges and problems and ultimately further their development.

The book beautifully explains how emergence is a process that moves from low-level rules to higher-level sophistication and how our bodies, our minds and the world we create and live in are all ruled by this phenomenon. Order is created from the bottom up, not from the top down. Johnson is not the first to document this, and it is surprising to read that his predecessors in such various fields as economist Adam Smith, philosopher Friedrich Engels and mathematician Alan Turing have all contributed in important ways to this idea. Nevertheless, decision making in politics and economics is often still from the top, and so clearly the concept of emergence is much less popular than it deserves. But this book has the potential to raise wider interest in this fundamental principle, which, if used wisely, could help us to shape our future for the better.

Jürgen Tautz is at the University of Würzburg, Germany.

E-mail: tautz@biozentrum.uni-wuerzburg.de

DOI: 10.1093/embo-reports/kvf085 